\title{
EVALUACIÓN IN VITRO DE LA EFICACIA DEL LOCALIZADOR APICAL ROOT ZX EN PRESENCIA DE HIPOCLORITO DE SODIO COMBINADO CON RC-PREP O EDTA COMPARADO AL MICROSCOPIO ELECTRÓNICO DE BARRIDO
}

\author{
${ }^{1}$ Monique Marie Gay Ortiz, ${ }^{2}$ Martha Elena Varón Plata, ${ }^{2}$ Oscar Gustavo Serrano Navas \\ ${ }^{1}$ Odontóloga, U. Javeriana, Residente II año, posgrado de Endodoncia, U. Santo Tomás. ${ }^{2}$ Odontólogos, \\ Residentes II año posgrado de Endodoncia, U. Santo Tomás. \\ Autor responsable de la correspondencia: Dra. Monique Marie Gay Ortiz \\ Correo electrónico: mmtgo@hotmail.com
}

\begin{abstract}
RESUMEN
Objetivo: Evaluar la eficacia del localizador Root ZX para determinar la Constricción Apical en presencia de hipoclorito de sodio al $5.25 \%$ Clorox ${ }^{\circledR}$ combinado con Rc- prep. Premier ${ }^{\circledR}$ y el hipoclorito de sodio al 5.25\% Clorox ${ }^{\circledR}$ combinado con EDTA al 10\% Eufar ${ }^{\circledR}$ en dientes uniradiculares.

Materiales y métodos: Se realizó un estudio experimental in vitro con 12 dientes uniradiculares divididos aleatoriamente en tres grupos: Grupo $1(\mathrm{n}=12)$ hipoclorito de Sodio al 5.25\% Clorox ${ }^{\circledR}$; Grupo $2(\mathrm{n}=6)$ hipoclorito de Sodio al 5.25\% Clorox ${ }^{\circledR}$ combinado con Rc- prep. Premier ${ }^{\circledR}$; Grupo $3(n=6)$ hipoclorito de Sodio al 5.25\% Clorox $^{\circledR}$ combinado con EDTA al 10\% Eufar $^{\circledR}$. Se utilizó el Root ZX para localizar la Constricción Cemento Dentina Conducto (CDC). Posteriormente, las muestras fueron divididas longitudinalmente, cubiertas con oro y observadas en el Microscopio Electrónico de Barrido (SEM), para corroborar la eficacia del instrumento en la localización de la CDC.

Resultados: Se encontraron diferencias estadísticamente significativas entre los dos instrumentos de medición (Root ZX y SEM) y para cada grupo de tratamiento. Con el hipoclorito de sodio y Rc - Prep, se obtuvo una medida más larga, en comparación con la obtenida con el SEM; una diferencia de $0.788 \mathrm{~mm}$. Por el contrario, el grupo del hipoclorito de sodio al 5.25\% con EDTA al 10\% expresó una medida más corta con relación a la obtenida en el SEM, dando una diferencia estadísticamente significativa de $-0.766 \mathrm{~mm}$. Conclusiones: Se podría sugerir que el Root ZX es eficaz en la localización de la Constricción Apical con un nivel de tolerancia de $+/-1 \mathrm{~mm}$. [Gay MM, Varón ME, Serrano OG. Evaluación in vitro de la eficacia del localizador apical Root ZX en presencia de hipoclorito de sodio combinado con Rc Prep o EDTA comparado con el Microscopio Electrónico de Barrido. Ustasalud Odontología 2003; 2: 97 - 106]
\end{abstract}

PALABRAS CLAVE: Root ZX, constricción apical, hipoclorito de sodio.

In vitro evaluation of the effectiveness of the apical locator Root $\mathrm{ZX}$ in presence of sodium hypochlorite combined with Rc - Prep or Edta compared with scanning electron microscope

\begin{abstract}
Purpose: To assess the effectiveness of the Root $\mathrm{Zx}$ to determine the Cement - Dentin - Canal junction $(C D C)$ in the presence of

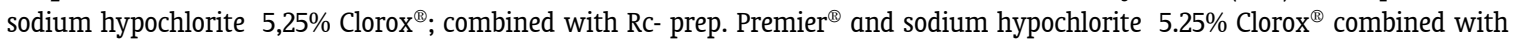
EDTA $10 \%$ Eufar $^{\circledR}$ in uniradicular teeth.

Material and methods: An experimental study in vitro with 12 uniradicular teeth was done. They wered divided in three groups: Grupo $1(\mathrm{n}=12)$ sodium hypochlorite $5,25 \%$ Clorox ${ }^{\circledR}$; Group $2(\mathrm{n}=6)$ sodium hypochlorite $5.25 \%$ Clorox ${ }^{\circledR}$ combined with Rc- Prep. Premier ${ }^{\circledR}$; Group $3(\mathrm{n}=6)$ sodium hypochlorite $5,25 \%$ Clorox $^{\circledR}$ combined with EDTA 10\% Eufar ${ }^{\circledR}$. Root ZX was used to locate the CDC; the samples were divided longitudinally, covered with gold and observed at the SEM, to corroborate the effectiveness of the instrument in the location of the $\mathrm{CDC}$.

Results: The results demonstrate statistically significant differences between Root ZX and SEM in each group. In group 1, a longer measurement was obtained with the Root ZX compared with the SEM, the difference was $0.788 \mathrm{~mm}$. On the other hand, in the group of the sodium hypochlorite 5,25\% with EDTA $10 \%$, a shorter measurement was obtained, $(-0.766 \mathrm{~mm})$ a statistically significant difference.

Conclusions: The results suggest that the Root ZX is effective in the location of the apical constriction with a level of tolerance of $+/-1 \mathrm{~mm}$.
\end{abstract}

KEY WORDS: Root ZX, Cement-Dentin-Canal junction (CDC), sodium hypochlorite, Rc - Prep, EDTA. 


\section{INTRODUCCIÓN}

La constricción apical localizada en cemento y/o dentina, corresponde al punto más estrecho y con menor riego sanguíneo en el interior del conducto. Desde el punto de vista biológico la Constricción Cemento - Dentina Conducto (CDC) se constituye como el accidente morfológico más relevante en cuanto a la longitud de trabajo se refiere, porque permite la adecuada conformación de un tope para la condensación del material de obturación, favorece el selle apical y permite el control del proceso inflamatorio gracias a la presencia del riego sanguíneo funcional en la zona apical inmediatamente adyacente a ésta. ${ }^{1}$

Esta constricción apical, generalmente coincide con la zona de transición entre la dentina y el cemento. Idealmente, un tratamiento de endodoncia se realiza en el complejo dentino-pulpar y su objetivo es tratar el conducto radicular rodeado por dentina, para que los cementoblastos que recubren el cemento, contribuyan a un cierre apical biológico, que es finalmente, el objetivo del tratamiento. Shilder, mencionó que el único punto de referencia que existe para establecer una adecuada longitud de trabajo es el ápice radiográfico; ${ }^{1-2}$ éste se determina empleando el método radiográfico. Sin embargo, presenta algunas desventajas como la superposición de las estructuras anatómicas que proporciona la imagen bidimensional de un objeto tridimensional, los riesgos de la radiación,, 4 pero, su principal desventaja es que no detecta la constricción apical, sólo se puede observar con exactitud el ápice radiográfico que se sabe se encuentra entre 0,5 y 1,5 mm de la CDC. ${ }^{1}$ Kuttler, demostró que este límite anatómico se encontraba localizado entre $0.524 \mathrm{y}$ $0.659 \mathrm{~mm}$ coronal al foramen apical. ${ }^{5}$

Los primeros localizadores apicales fueron desarrollados en el año de 1942 por Suzuki, ${ }^{6-8}$ posteriormente en 1962, Sunada utilizó un dispositivo de corriente directa en un circuito simple y encontró la constante de la resistencia eléctrica para el ligamento periodontal y la membrana mucosa con un valor de $(6,5 \mathrm{k0}) .{ }^{1,9-14}$ Actualmente, se encuentra en el mercado la tercera generación de localizadores apicales o de doble frecuencia, que miden la impedancia con dos frecuencias eléctricas distintas; dentro de éstos se encuentra el Root ZX. Diversos estudios in vivo e in vitro han probado la eficacia de este localizador para determinar la constricción apical o forámen en presencia de soluciones anestésicas, irrigantes y quelantes entre otras. ${ }^{1,3}$
En 1996, Shabahang estableció que el Root ZX posee una confiabilidad del $96.2 \% .^{10}$ En un estudio in vitro realizado por Weiger en 1999, se compararon dos localizadores tipo frecuencia Root ZX (J. Morita, Tokio, Japan) y el Apit (Osada Electric Co., Ltd, Tokio, Japan) y se concluyó que el Root ZX fue el más preciso, en un rango entre $0.76 \mathrm{~mm}$ y $0.85 \mathrm{~mm}$ en la lectura de medición APEX y 0.5 respectivamente, con un nivel de tolerancia de $+/-0.5 \mathrm{~mm}$. 15 Jenkins y colaboradores realizaron un estudio para probar la eficacia del localizador Root ZX en presencia de una gran variedad de irrigantes, comúnmente utilizados, durante el tratamiento de endodoncia; los resultados mostraron un $81 \%$ de eficacia en ausencia de hipoclorito de sodio, un $83 \%$ en presencia de un hipoclorito de sodio al $2.25 \%$ y un $85 \%$ con un hipoclorito de sodio al $5.25 \%$. $^{8}$

Sin embargo, estas sustancias han sido probadas de manera individual dentro del conducto, y es importante tener en cuenta que durante la limpieza y la conformación, se hace necesaria la combinación de una solución irrigante con un agente quelante para eliminar la capa de barrillo dentinal, exponer los túbulos dentinales y de esta manera facilitar la remoción de detritus, bacterias y endotoxinas que se encuentran presentes dentro del sistema radicular de conductos. Esto es poco probable utilizando solamente una sustancia irrigadora como el hipoclorito de sodio. La combinación de ésta con agentes quelantes como el Rc Prep. y el EDTA permitirá comprobar la eficacia del localizador apical Root ZX para determinar la constricción apical.

El objetivo de este estudio fue evaluar la eficacia del localizador Root ZX para determinar la constricción apical en presencia de hipoclorito de sodio 5.25\% Clorox $^{\circledR}$ combinado con Rc - Prep. Premier ${ }^{\circledR}$ e hipoclorito de sodio 5.25\% Clorox $^{\circledR}$ combinado EDTA 10\% Eufar ${ }^{\circledR}$ en dientes uniradiculares.

\section{MATERIALES Y MÉTODOS}

Se realizó un estudio de carácter experimental in vitro. Se seleccionó una muestra por conveniencia constituida por 12 dientes uniradiculares superiores y/o inferiores que cumplieron con los criterios de inclusión. El tamaño de la muestra obedeció a criterios técnicos y económicos, puesto que las mediciones obtenidas con el localizador apical fueron comparadas con las mediciones establecidas a partir de la prueba de referencia, es decir el microscopio electrónico de barrido (SEM). Los criterios de inclusión 
tenidos en cuenta fueron los siguientes: Dientes permanentes uniradiculares superiores y/o inferiores extraídos con fines ortodónticos y/o periodontales (previa firma de la carta de consentimiento informado por parte del paciente) con formación radicular completa, libre de caries radicular, sin perforaciones a nivel cervical medio o apical, reabsorción interna, externa superficial, inflamatoria o por reemplazo, diente sin cirugía periradicular, abrasiones previas y/o restauraciones metálicas extensas. Los criterios de exclusión fueron, dientes temporales, biradiculares o multiradiculares.

Los dientes fueron lavados con agua y jabón, almacenados en agua destilada y distribuidos aleatoriamente en tres grupos, que corresponde a una variable categórica medida en escala nominal. Grupo 1 ( $n=12$ dientes): Grupo control, irrigación inicial con hipoclorito de sodio al 5.25\% Clorox $^{\circledR}$. Grupo 2 ( $\mathrm{n}=6$ dientes): se escogieron aleatoriamente seis dientes del Grupo 1 y se realizó una segunda irrigación con hipoclorito de sodio al 5.25\% Clorox ${ }^{\circledR}$ más un agente quelante, Rc-prep. Premier ${ }^{\circledR}$. Grupo 3 ( $n=6$ dientes): los seis dientes restantes del Grupo 1 , se les realizó una segunda irrigación con hipoclorito de sodio al 5.25\% Clorox $^{\circledR}$ más un agente quelante EDTA al $10 \%$ Eufar $^{\circledR}$.

La eficacia, del Root ZX fue definida como la menor diferencia entre los grupos de tratamiento en comparación con la medida obtenida con el microscopio electrónico de barrido (SEM), sea 0 no estadísticamente significativa.

La investigación fue realizada bajo todos los principios científicos y éticos necesarios en pro del desarrollo de la ciencia para lo cual se elaboró un consentimiento informado y voluntario de los pacientes, autorizando su participación en el estudio; según el título II artículo 11 de la Resolución No 008430 del 04 de octubre de 1993, expedida por el Ministerio de Salud de la República, se clasifica como de Riesgo Mínimo.

Las coronas de los dientes seleccionados, fueron cortadas con un disco de diamante a nivel de la unión Amelo Cementaria (UAC) por un solo operador, para simplificar el acceso al conducto radicular y obtener un punto de referencia confiable y seguro. Los dientes fueron sujetados con una pinza 150 y colocados en contacto con un gel conductivo que simuló el periodonto. Este gel fue descrito por Donnelly en 1993 y consistió en gelatina (Royal ${ }^{\circledR}$ sin sabor) con solución de sodio clorhídrico al 0.9\%, 16 en una caja de acrílico de 10 × 3 × 3 con su respectiva tapa según
Kaufman y Katz 17 (Foto 1). Las tapas tenían un orificio para introducir el diente; una vez en posición, cada diente fue pegado a la superficie con pegante instantáneo $\left(\right.$ Pegadit $\left.^{\circledR}\right)$ y posteriormente se procedió a la preparación del gel conductivo. Se colocó la tapa sobre la caja de acrílico hasta que gelificó.

La medida eléctrica fue obtenida usando el localizador apical Root ZX (J. Morita MFG Corp. Kyoto, Japan). La medida en el Grupo 1 fue tomada después de la irrigación de $2 \mathrm{ml}$ de hipoclorito de sodio al 5.25\% Clorox ${ }^{\circledR}$ dentro del conducto radicular, el cual no había sido preparado, instrumentado o secado antes de tomar la medida con el localizador apical (Foto 2). La irrigación se realizó con una jeringa de $5 \mathrm{cc}\left(\right.$ Ultradent $^{\circledR}$ ) y una aguja endo - EZE calibre 27 de la misma casa comercial.

La longitud fue tomada con una lima $\mathrm{K} 0.10$ nueva (Maillefer $\left.{ }^{\circledR}\right)$ con doble tope para mayor seguridad. El localizador apical Root ZX fue utilizado, de acuerdo con el manual de instrucciones que proporciona la casa fabricante (J. Morita MFG Corp. Kyoto. Japan). Éste menciona que cuando la señal alcanzara la marca "APEX", la lima sería sacada hasta que en la pantalla se alcance la marca "0.5" (Foto 3).

Posteriormente, se retiró la lima y se llevó a una regla milimetrada nueva (Maillefer ${ }^{\circledR}$ ) (Foto 4) para registrar la longitud de trabajo que fue dada en $\mathrm{mm}$. La medida de precisión fue de $0.25 \mathrm{~mm}$. según Ounsi y colaboradores 18 que sugieren que cuando la punta del instrumento A está un poco corta con relación al punto $\mathrm{C}$ o justo por delante del punto $\mathrm{B}$, se considera que la longitud será $\mathrm{B}+0.75$ $\mathrm{mm}$., de la misma manera, si la longitud está justo por delante de $\mathrm{B}$ o un poco corta de $\mathrm{B}+0.5 \mathrm{~mm}$. se considera que $\mathrm{B}+0.25$ esto equivale a decir que clínicamente esta longitud es X5 + mm. o X .5 mm. ${ }^{5}$ Al siguiente día se tomó la medida en el grupo 2, después de una irrigación de $2 \mathrm{ml}$ de hipoclorito de sodio al 5.25\% Clorox ${ }^{\circledR}$ y de llevar una lima $\mathrm{K}$ \# 10 nueva (Maillefer ${ }^{\circledR}$ ) con agente quelante Rc - Prep Premier ${ }^{\circledR}$ (Foto 5). El resto del procedimiento fue igual al del grupo 1 . Al tercer día se tomó la medida en el grupo 3, después de una irrigación con $2 \mathrm{ml}$ de hipoclorito de sodio al 5.25\% Clorox $^{\circledR}$ y de llevar con una lima $\mathrm{K} \# 10$ nueva EDTA al 10\% Eufar ${ }^{\circledR}$ dentro del conducto antes de la tomar la medida con el localizador apical (Foto 6). El resto del procedimiento fue el mismo de los grupos 1 y 2. 


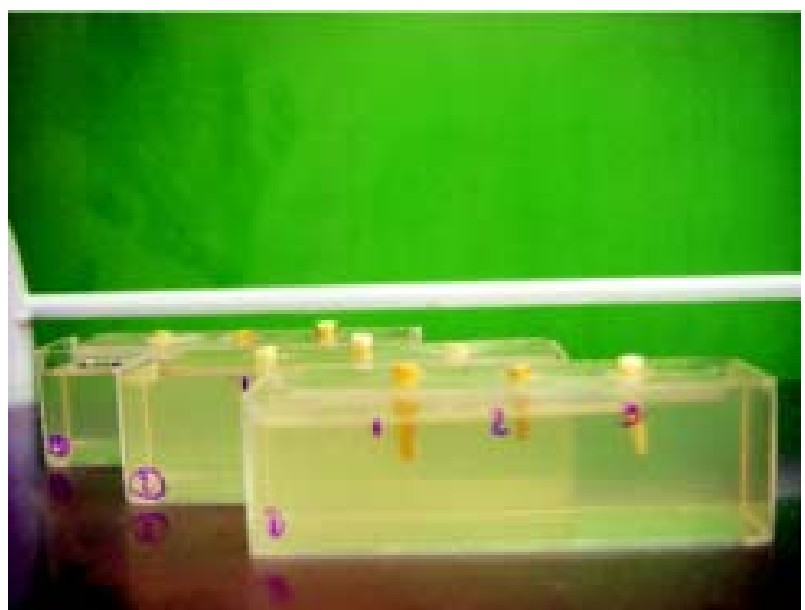

Foto 1. Cubos de acrílico de 10 x 3 x 3 descrito por Kaufman y Katz. ${ }^{17}$

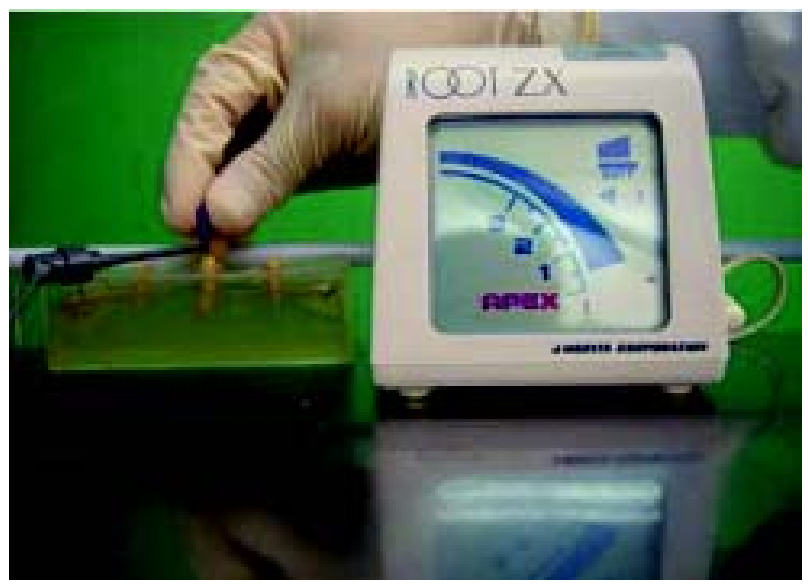

Figura 3. Toma de la longitud de trabajo con lima $\mathrm{K} \# 10$ (Maillefer Suiza ${ }^{\circledR}$ ).

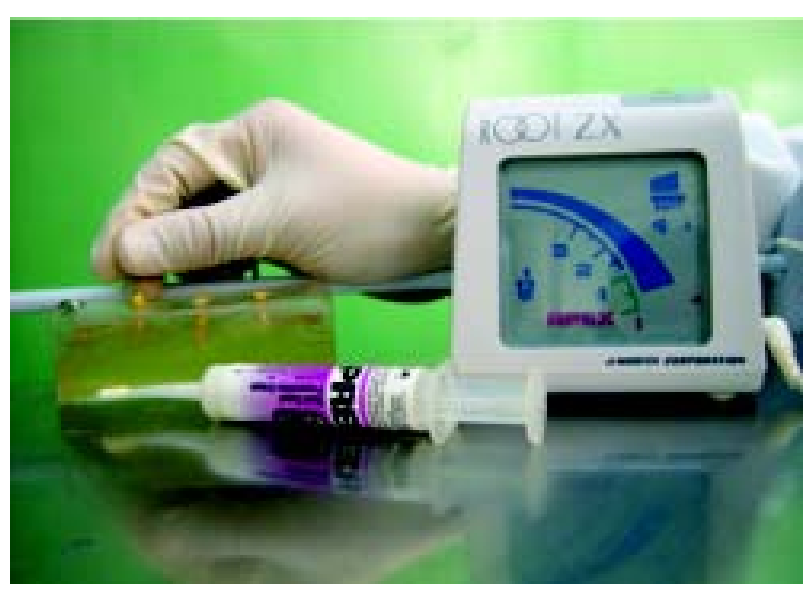

Foto 5. Toma de la longitud de trabajo con lima K \# 10 (Maillefer Suiza ${ }^{\circledR}$ ) previa irrigación con $2 \mathrm{ml}$ de hipoclorito de sodio. 100

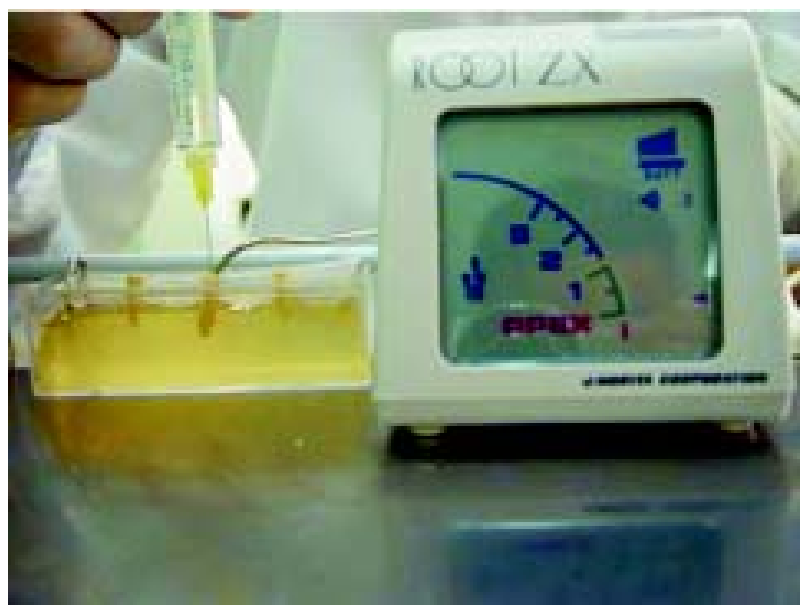

Foto 2. Irrigación del conducto con $2 \mathrm{ml}$ de hipoclorito de sodio al $5.25 \%$ Clorox $^{\circledR}$.

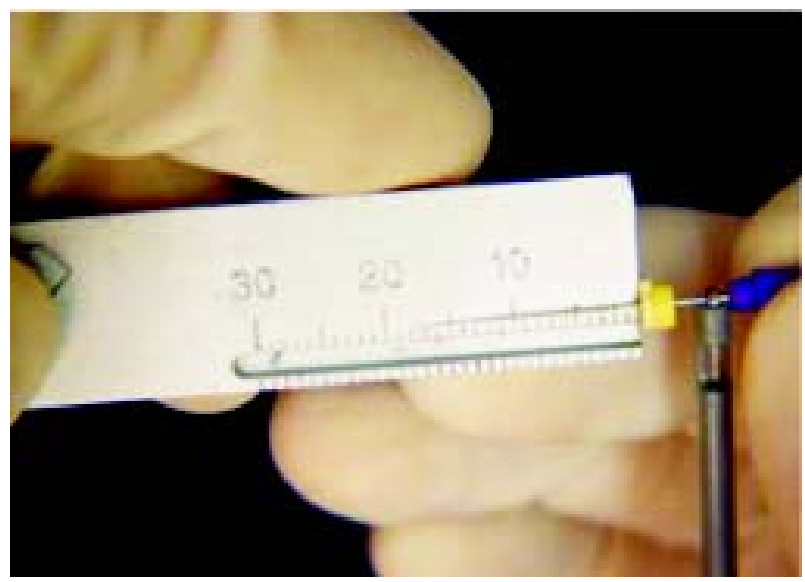

Foto 4. Registro de longitud de trabajo en mm sobre una regla milimetrada (Maillefer ${ }^{\circledR}$ Suiza).

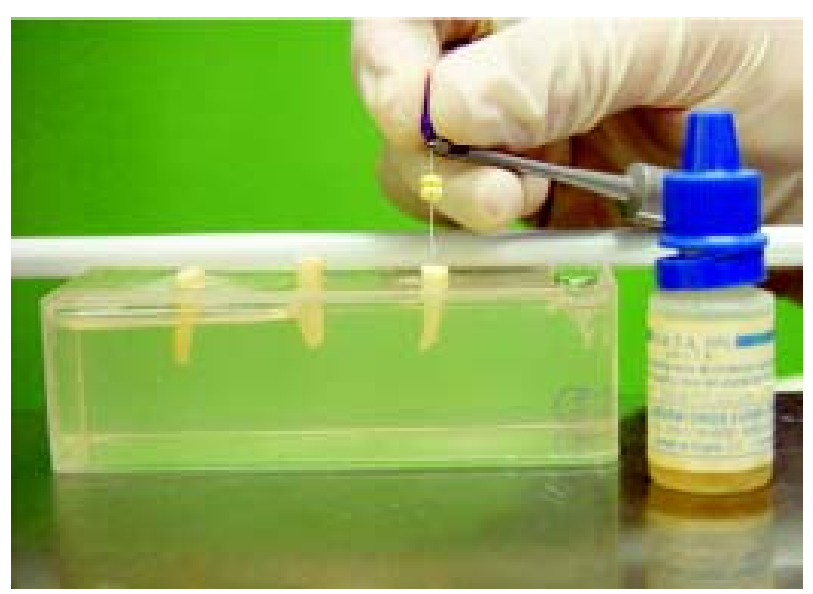

Foto 6. Toma de la longitud de trabajo con lima K \# 10 (Maillefer Suiza ${ }^{\circledR}$ ) previa irrigación con $2 \mathrm{ml}$ de hipoclorito de sodio. 


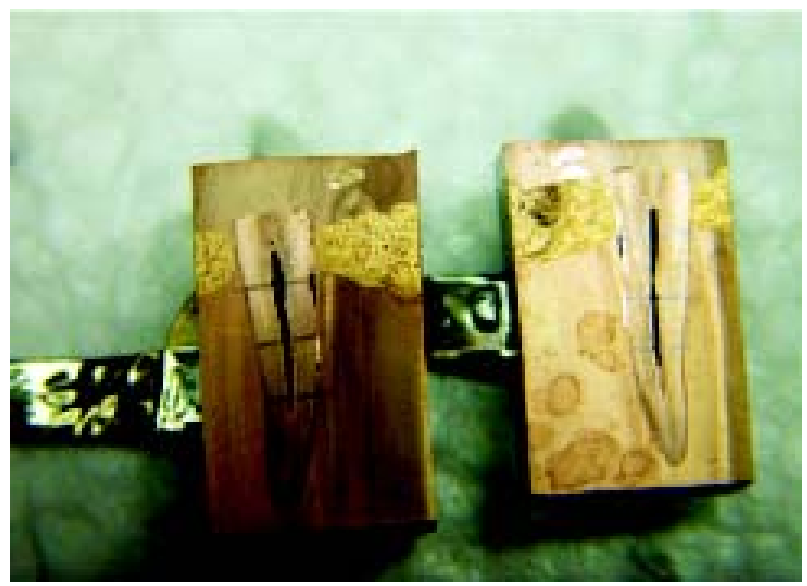

Foto 7. Muestras recubiertas con oro, listas para ser observadas al SEM.

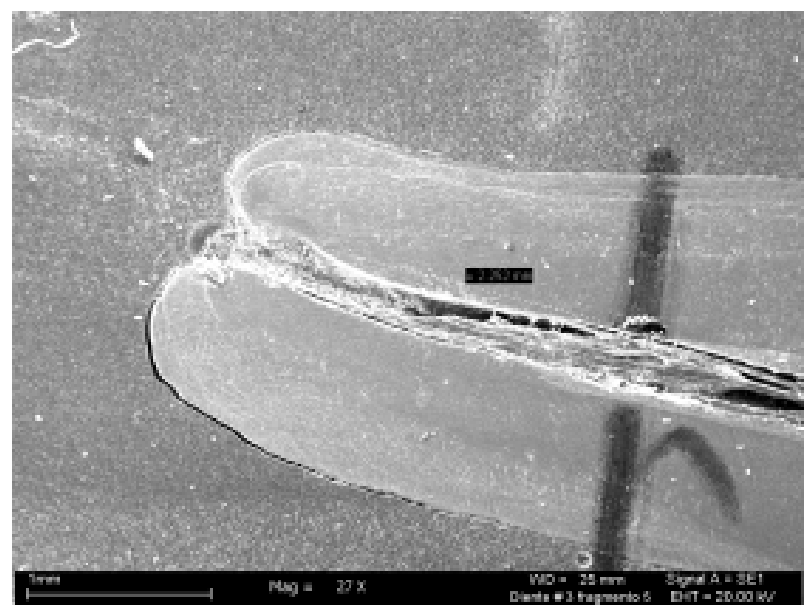

Foto 8. Fotografía CD con SEM a 27X .

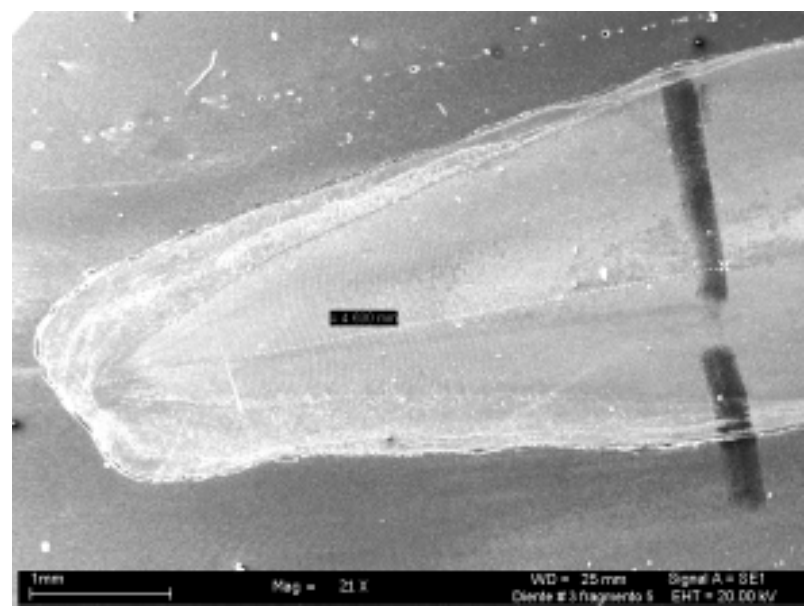

Foto 9. Fotografía CD con SEM a 27X.
La medida se repitió tres veces en cada grupo y en cada diente; los instrumentos utilizados fueron nuevos y se utilizaron dos topes en cada lima para evitar el movimiento accidental de éstos durante el proceso de medición, todo el proceso fue realizado por un solo operador.

Posteriormente, se realizó la preparación de las muestras para ser observadas en el Microscopio Electrónico de Barrido (SEM) LEO Modelo $1450 \mathrm{VP}^{\circledR}$. Primero, se marcó con un lapicero el sitio del corte longitudinal, luego se realizó un lavado externo de los dientes con agua destilada y jabón neutro para controlar la grasa, después, los dientes fueron montados en un molde plástico con resina epóxica transparente más un catalizador (Buettler ${ }^{\circledR}$ ) a $40^{\circ} \mathrm{C}$ durante 12 horas, para conseguir una adecuada polimerización. Se desmoldó y se realizó el corte longitudinal en la máquina Isomet ${ }^{\circledR}$, se utilizó un disco diamantado de tres pulgadas y refrigeración con agua destilada; se obtuvieron dos muestras por cada diente para un total de 24, éstas fueron observadas en el microscopio binocular Nikon SMZ-10 ${ }^{\circledR}$, (Nikon Corporation, Tokio, Japan) para controlar las huellas o marcas en la superficie. Por último, se realizó un pulido con lija número 600 , paños de nylon y alúmina en la pulidora Ecomet ${ }^{\circledR}$. Todas las muestras $(n=24)$ fueron nuevamente numeradas con una cinta de aluminio de acuerdo a las especificaciones del Instituto Colombiano de Petróleos (ICP); se colocó un nuevo número para identificar cada diente, un lado de la muestra fue marcado como Vd y el otro como Vda. Posteriormente, fueron colocadas en un porta muestras, pegadas con grafito en Isopopanol de la EMS Electric Microscopio Science $^{\circledR}$, y se llevaron a un recubridor de muestras Eduards S150 ${ }^{\circledR}$ para ser recubiertas con oro. Los trazos se realizaron a lápiz en diferentes niveles de las muestras con el fin de obtener varias medidas en una misma raíz en el microscopio electrónico de barrido (SEM) LEO modelo $1450 \mathrm{VP}^{\circledR}$ que utiliza el software LEO $32^{\circledR}$, y así, calcular la distancia entre el punto de referencia coronal y la CDC (Foto 7).

Las muestras fueron observadas y fotografiadas con una magnificación de 27X, sin embargo en algunos casos, en los que la longitud radicular seguía siendo demasiado grande, se disminuyó a $22 \mathrm{X}$ e inclusive hasta $16 \mathrm{X}$ (Foto 8, 9).

Con el fin de eliminar el mayor número de variables posibles y estandarizar la muestra, se escogieron dientes con características radiculares morfológica y anatómicamente similares, y se realizó un enmascara-

101 
miento doble para el operador, con relación a las sustancias utilizadas para irrigación del conducto. La medida final con el SEM fue tomada en consenso con los 3 investigadores debido a la dificultad para visualizar exactamente la ubicación de la CDC.

Inicialmente, se describieron los promedios de los triplicados de las medidas obtenidas con el localizador apical y el SEM por separado para cada grupo de tratamiento y se compararon aplicando un análisis de ANOVA de una vía. Además, se estableció el nivel de precisión para cada uno, calculando el intervalo de confianza del 95\%. ${ }^{19}$ Posteriormente, se evaluaron las diferencias en los promedios por grupo de tratamiento para cada instrumento, y se aplicó, también, un análisis de varianza ANOVA de una vía. ${ }^{19}$ La comparación entre los dos instrumentos, se realizó aplicando una prueba t de Student apareada y por separado para cada grupo de tratamiento. ${ }^{19}$ Finalmente, se estableció el nivel de acuerdo entre los dos instrumentos, aplicando la metodología de Bland y Attman que permitió obtener además los límites de acuerdo entre las dos mediciones para cada grupo de tratamiento. ${ }^{20}$

Todo el análisis consideró un nivel de significancia a 0,05. La base de datos se digitó en Excel y el análisis se elaboró en STADA 8.0 La eficacia se determinó con base en la menor diferencia en comparación con la medida obtenida con el microscopio electrónico de barrido.

\section{RESULTADOS}

Al evaluar los promedios de las diferencias obtenidas para cada grupo de tratamiento, no se detectaron diferencias estadísticamente significativas ( $\mathrm{p}>0.95$ ), lo cual permitió establecer el promedio de las mediciones, el error estándar y el intervalo de confianza del 95\% para cada grupo de tratamiento. En forma similar a las mediciones obtenidas con el localizador apical no se detectaron diferencias estadísticamente significativas entre los promedios de las mediciones para el SEM ( $p>0.6$ ) (Tabla 1).

Considerando que el análisis del microscopio implicaba el corte longitudinal de cada diente, inicialmente, se presenta el análisis comparativo de las dos muestras para cada diente y por grupo de tratamiento.

Los resultados de la comparación de los promedios por muestra para cada grupo de tratamiento se muestran en la Tabla 2. El análisis permite concluir que no hay una diferencia estadísticamente significativa entre los promedios, por lo cual el promedio de las dos caras se considera la base de la medición de la reproducibilidad con el SEM para cada grupo de tratamiento.

Al comparar los promedios obtenidos con el localizador apical por grupo de tratamiento no se encontraron diferencias estadísticamente significativas $(\mathrm{p}=0.60)$ (Figu-

Tabla 1. Precisión de las mediciones obtenidas con el localizador apical y el SEM por cada grupo de tratamiento.

\begin{tabular}{c|c|c|c|c|c}
\hline $\begin{array}{c}\text { Tipo de } \\
\text { instrumento }\end{array}$ & Grupo & Promedio & Error Estándar & \multicolumn{2}{|c}{ Intervalo de confianza 95\% } \\
\hline \multirow{2}{*}{$\begin{array}{c}\text { Localizador } \\
\text { apical }\end{array}$} & 1 & 14.89 & 0.37 & 14.13 & 15.64 \\
\cline { 2 - 6 } & 2 & 15.17 & 0.68 & 13.74 & 16.59 \\
\cline { 2 - 6 } & 3 & 13.91 & 0.34 & 13.19 & 14.66 \\
\hline \multirow{2}{*}{ SEM } & 2 & 14.38 & 0.81 & 12.57 & 16.18 \\
\cline { 2 - 6 } & 3 & 14.62 & 0.37 & 13.84 & 15.41 \\
\hline
\end{tabular}

Tabla 2. Comparación de los promedios de las medidas obtenidas por muestra para cada grupo de tratamiento.

\begin{tabular}{c|c|c|c}
\hline Grupo & Muestra & Promedio $(\mathrm{mm})$. & Probabilidad \\
\hline \multirow{2}{*}{2} & Vd & 14.295 & \multirow{2}{*}{0.93} \\
\cline { 2 - 3 } & Vda & 14.45 & \multirow{2}{*}{0.595} \\
\cline { 2 - 3 } 3 & Vd & 14.42 & 14.82 \\
\hline
\end{tabular}


ra 1). Igual fenómeno se detectó al comparar los promedios de las mediciones obtenidas con el SEM por grupo de tratamiento (grupo 2 y grupo 3), ( $p=0.84$ ) (Figura 2). Los resultados de la t Student 19 apareada muestran diferen cias estadísticamente significativas entre los dos instrumentos de medición y para cada grupo de tratamiento. Los resultados se presentan en la Figura 3.

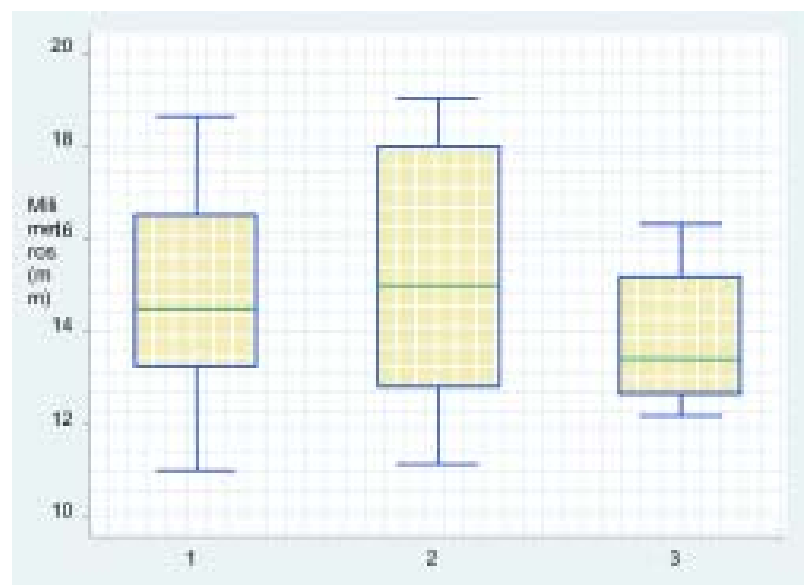

Figura 1. Mediciones con Root ZX por grupo.

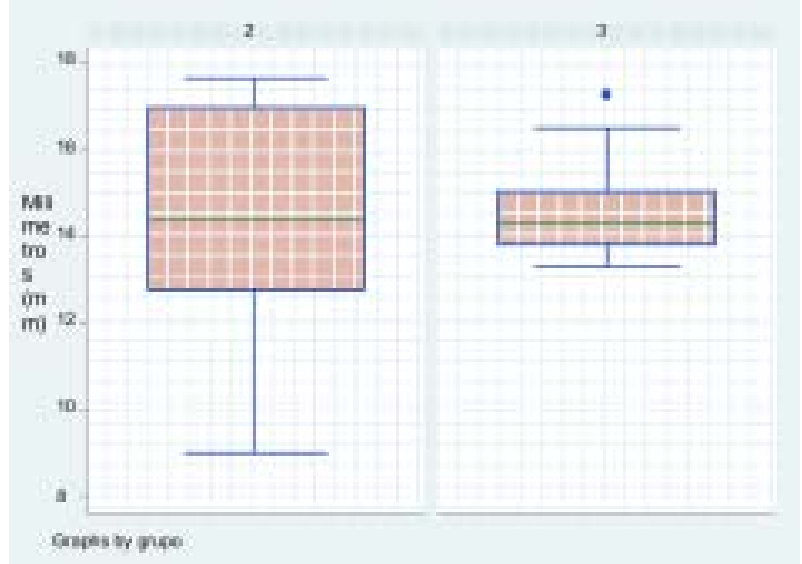

Figura 2. Comparación de las mediciones con SEM por grupo.

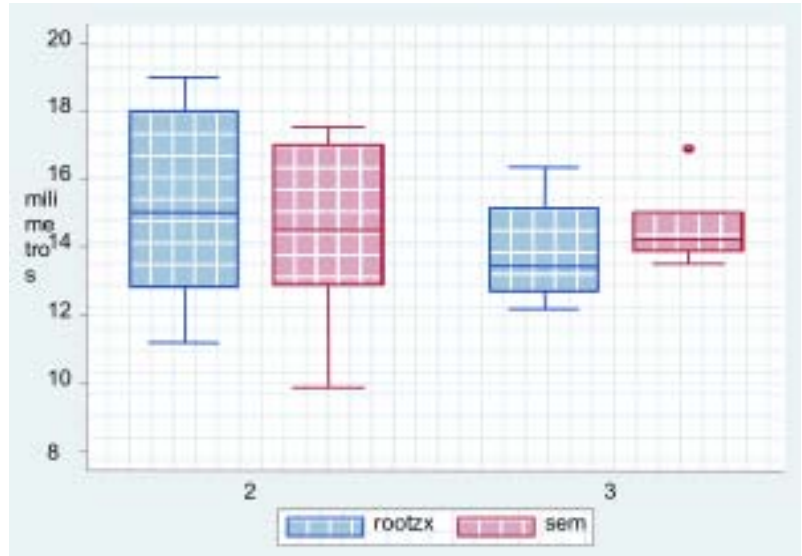

Figura 3. Mediciones con los dos instrumentos por grupo.
La metodología de Bland y Altman 20 estableció un nivel promedio de diferencias entre los dos instrumentos. El promedio de las diferencias entre el localizador apical y el SEM fue positivo, $0.79 \mathrm{~mm}$, con una desviación estándar de $0.625 \mathrm{~mm}$ para el grupo 2. Para el grupo 3 el promedio fue negativo $-0.77 \mathrm{~mm}$, con una desviación estándar de $0.65 \mathrm{~mm}$. Estos datos permitieron establecer los límites de acuerdo; para el grupo 2, el límite inferior fue -0.437 $\mathrm{mm}$ y el límite superior $2.01 \mathrm{~mm}$; para el grupo 3, el límite inferior fue $-2.04 \mathrm{~mm}$ y el límite superior $0.505 \mathrm{~mm}$ (Figura 4).
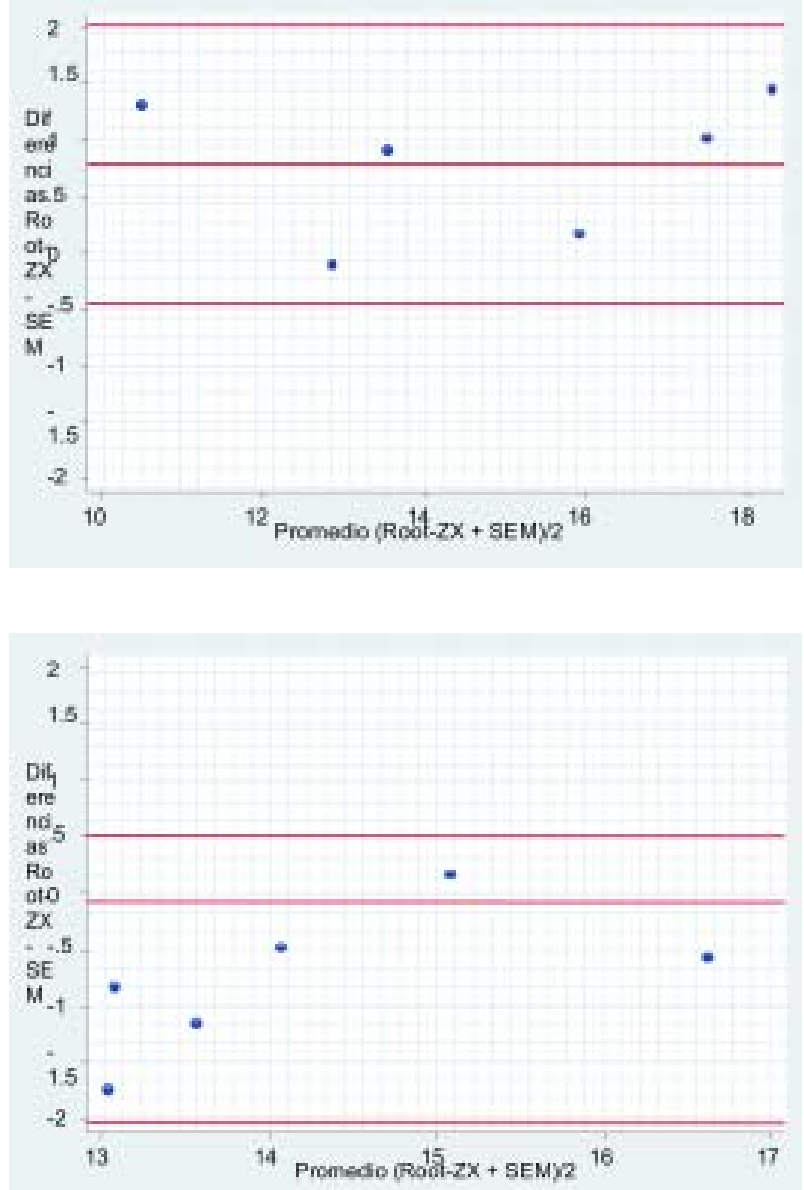

Figuras 4. Límites de acuerdo entre el Root ZX y el SEM por grupo.

\section{DISCUSIÓN}

La unión cemento dentina conducto o CDC y el foramen apical son dos puntos de referencia frecuentemente empleados para determinar el límite apical de la instrumentación y posterior obturación del conducto. ${ }^{21}$ 
El localizador es un instrumento altamente eficaz para ubicar la unión cemento - dentina - conducto. Éste corresponde al punto en el cual dos tejidos, cemento y dentina, convergen dentro del conducto radicular. Este punto es susceptible a modificaciones dependiendo de cada situación clínica en particular y a la extensión del cemento dentro de la raíz. El foramen apical no es una referencia anatómica confiable para ser usada como límite durante la preparación; su uso como tope puede causar lesiones en la región periapical. ${ }^{21}$ Schilder mencionó que el único punto de referencia que existe para establecer una adecuada longitud de trabajo es el ápice radiográfico. ${ }^{1,2}$

La radiografía ha sido utilizada durante muchos años para determinar este límite apical durante la preparación., 22 Sin embargo, este método tiene limitaciones debido a que obtiene una imagen bidimensional de un objeto que realmente es tridimensional, superposición de las estructuras anatómicas, riesgo de radiación, no permite detectar la posición del forámen en el conducto radicular, ${ }^{4,23}$ no localiza la CDC, sólo permite observar el ápice radiográfico, el cual estadísticamente se encuentra entre $0.5-1 \mathrm{~mm}^{3}$ El localizador apical permite una adecuada localización de esta referencia anatómica. ${ }^{13,18,}$ ${ }^{2426}$ Ounsi y colaboradores reportaron, en 1.998, que el método radiográfico tiene un $97.06 \%$ de eficacia en la localización del foramen apical y que el localizador apical tiene una precisión del $84.46 \%$ para detectar la CDC. ${ }^{27} \mathrm{~A}$ pesar de que con el método radiográfico se obtiene un alto porcentaje de precisión, éste varía según el operador y la técnica utilizada para la toma de la radiografia (paralelismo o bisectriz). Por el contrario, el localizador apical no se altera por variaciones intra e interoperatorias, lo que permite la reproducibilidad de los resultados.

Los resultados de este estudio demuestran la reproducibilidad de las medidas obtenidas con el Root ZX $(\mathrm{p}>0.95)$.

El microscopio electrónico de barrido permite una orientación adecuada de la muestra examinada y una identificación de las estructuras de referencia anatómicas. 28 En este estudio el análisis de precisión con el SEM para cada grupo de tratamiento no detectó diferencias estadísticamente significativas entre los promedios de las mediciones ( $p>0.6)$.

Durante un tratamiento de conducto es esencial la irrigación con sustancias químicas y quelantes como el hipoclorito de sodio al 5.25\% Clorox $^{\circledR}$, Rc - Prep Premier ${ }^{\circledR}$ y EDTA al 10\% Eufar ${ }^{\circledR}$, entre otros, como complemento en el proceso de limpieza y conformación antes de realizar la obturación tridimensional del conducto. Su objetivo es promover el arrastre, la lubricación y la disolución del tejido orgánico, además de actuar sobre la flora microbiana presente y eliminar la capa de barrillo dentinal. ${ }^{29}$ Un estudio sobre localizadores apicales evaluó su eficacia en presencia de diversas soluciones irrigadoras como el alcohol, la solución salina al 0.9\%, el EDTA al 10\%, y el hipoclorito de sodio. Se concluyó que las soluciones no conductoras permiten mejor detección de la posición de la lima en relación con el foramen e intervienen menos con las restauraciones metálicas. ${ }^{30}$ Jenkins y colaboradores evaluaron la eficacia del Root ZX utilizando sustancias como la lidocaína al 2\% con epinefrina 1:100.000, el hipoclorito de sodio al 0.25\%, EDTA, Rc - Prep, Peridex, peróxido de hidrógeno al 3\%, y encontraron que su eficacia no se altera con el tipo de irrigante empleado. ${ }^{8}$ Weiger y colaboradores reportaron una alta eficacia del localizador apical Root ZX en presencia del hipoclorito de sodio al $1 \% .{ }^{15}$ Otros autores como Cemal utilizaron hipoclorito de sodio al 0.5\%, al 1\%, al 2.65\%, y al 5.25\% dentro del sistema de conductos radicular y demostraron que la eficacia del Root ZX no se afecta al aplicar estas sustancias en diferentes concentraciones. ${ }^{31}$ Después de una completa revisión de literatura, se observó que ningún estudio evaluaba la eficacia del localizador apical con la combinación de una sustancia irrigadora y un agente quelante, siendo éste un procedimiento de rutina durante la preparación de los conductos radiculares. Debido a esta carencia, se decidió evaluar el Root ZX con una mezcla de hipoclorito de sodio al 5.25\% con Rc- Prep y EDTA al $10 \%$. Al comparar los promedios obtenidos con el localizador apical por grupo de tratamiento no se encontraron diferencias estadísticamente significativas $(p=0.60)$. Esto fue corroborado al comparar los promedios de las mediciones obtenidas con el SEM por grupo de tratamiento $(\mathrm{p}=0.84)$.

Los resultados obtenidos demuestran diferencias estadísticamente significativas entre el instrumento de medición Root ZX, el SEM utilizado como instrumento para corroborar y/o comparar la medida del localizador apical y para cada grupo de tratamiento, con el hipoclorito de sodio al 5.25\% Clorox $^{\circledR}$ y Rc - Prep. Premier ${ }^{\circledR}$, se obtiene una medida más larga en comparación con la obtenida con el SEM, una diferencia de $0.788 \mathrm{~mm}$ estadísticamente significativa. Por el contrario, en el grupo del hipoclorito de sodio al 5.25\% Clorox $^{\circledR}$ con EDTA al 10\% Eufar $^{\circledR}$ se obtuvo una medida más corta con relación a la observada en 
el SEM, dando una diferencia estadísticamente significativa de $-0.766 \mathrm{~mm}$, esto puede ser debido a la interacción química de estas sustancias al ser mezcladas, alterando sus propiedades químicas.

El EDTA es un ácido débil que en solución establece un equilibrio de disociación en el que libera parcialmente iones hidronio $(\mathrm{H}+)$ que se neutralizan con los iones hidroxilo generados por el carácter alcalino del hipoclorito de sodio, lo que conllevaría a que el $\mathrm{pH}$ del medio sea de tendencia neutra, condición en la cual se favorece el ataque del EDTA a la dentina liberando iones calcio que son quelados con él, en una reacción de equilibrio. Los iones presentes en la solución, en estas condiciones aportan conductividad, haciendo que la corriente colocada al instrumento prefiera desplazarse a través de la solución. ${ }^{32}$

El localizador apical determina la ubicación de la constricción apical, midiendo la reducción de la resistencia cada milímetro, a medida que se aproxima a la CDC, encontrando que ésta se hace mínima en el punto en el que éste es alcanzado. La presencia de una solución iónica disminuiría la resistencia con lo cual la medida mínima se alcanzaría antes del punto deseado. ${ }^{32}$

En el caso del Rc - Prep, el peróxido de urea proporciona unas condiciones más bajas de $\mathrm{pH}$ que aceleran la liberación de iones calcio de la dentina. La presencia de más iones calcio en la solución obliga a que se de el equilibrio de la reacción entre EDTA y los iones calcio hacia la producción de más quelato EDTA - $\mathrm{Ca}$, con lo cual el número de iones en solución sería menor comparado con el primer caso. Además, hay una reacción del peróxido de urea con el hipoclorito de sodio en el que se genera oxígeno molecular que no aporta a la conductividad y iones hidróxilo que son neutralizados con los iones hidronio del ácido, disminuyendo el número de especies cargadas en solución. Esto conlleva a una menor conductividad, es decir, mayor resistencia al paso de la corriente y por consiguiente las medidas registradas por el localizador apical serían más largas. ${ }^{32}$

Con base en esto surgen las siguientes hipótesis, las cuales son importantes para futuras investigaciones: Las soluciones al ser combinadas modifican la resistencia del medio, la interacción de las sustancias con los iones Calcio de la dentina liberados durante la quelación afectan la conductividad eléctrica del Root ZX y el pH influye en la disociación iónica y por ende en la conductividad del medio.
Shabahang y colaboradores comprobaron la eficacia del Root ZX en dientes vitales sin ningún tipo de preparación, y reportaron una confiabilidad clínica del $96.2 \%$ para localizar el foramen, dentro de un rango de error de $+-0.5 \mathrm{~mm} .{ }^{10}$ Las medidas obtenidas dentro de este rango son consideradas altamente eficaces. Otros estudios, permiten un rango más amplio, hasta $1 \mathrm{~mm},{ }^{23}$ debido a que la zona apical, presenta una amplia variabilidad. De esta manera, el Root ZX podría ser considerado preciso en todos los casos. Con base en los resultados obtenidos, y aplicándolos a la práctica clínica, se sugiere que cuando se utilice hipoclorito de sodio y Rc - Prep se disminuya 1.0 $\mathrm{mm}$ a la longitud de la lima cuando la barra de lectura del Root ZX marque 0.5.

Se puede concluir, que el localizador apical Root ZX ${ }^{\circledR}$ y el microscopio electrónico de barrido (SEM) en las muestras de hipoclorito de sodio al 5.25\% Clorox $^{\circledR}$, hipoclorito de sodio al 5.25\% Clorox ${ }^{\circledR}$ combinado con Rc - Prep Premier ${ }^{\circledR}$ e hipoclorito de sodio al 5.25\% Clorox ${ }^{\circledR}$ combinado con EDTA al 10\% Eufar $^{\circledR}$ muestran reproducibilidad de los resultados, y que no existen diferencias estadísticamente significativas entre los tres diferentes grupos de tratamiento con el localizador apical Root ZX ${ }^{\circledast}$. La eficacia del localizador apical Root ZX ${ }^{\circledR}$ en el grupo de hipoclorito de sodio al 5.25\% Clorox $^{\circledR}$ combinado con Rc - Prep Premier ${ }^{\circledR}$ al ser comparada con la medida obtenida en el SEM tiende a dar medidas más largas, estadísticamente significativas y el grupo del hipoclorito de sodio al 5.25\% Clorox ${ }^{\circledR}$ combinado con EDTA al 10\% Eufar ${ }^{\circledR}$ al ser comparado con la medida obtenida en el SEM tiende a dar medidas más cortas, estadísticamente significativas.

Durante el análisis con el SEM, se tuvo dificultad para identificar con claridad la unión de los dos tejidos que convergen dentro del conducto, esto pudo suceder por el corte, por la extensión del cemento dentro de éste y/o por la dificultad en la diferenciación del cemento y la dentina. Por esta razón se recomienda la tinción de los tejidos para permitir una mejor y más fácil identificación de la CDC.

Igualmente, se recomienda realizar futuras investigaciones sobre los procesos químicos que ocurren al combinar varias sustancias dentro del conducto y como éstas pueden alterar la conductividad eléctrica variando la precisión del localizador apical. 


\section{BIBLIOGRAFÍA}

1. West J, Roane J. Sistema de limpieza y conformación de los canales radiculares. En: Cohen S, Burns R. Vías de la Pulpa. 7ma ed. Madrid: Editorial Harcourt- Mosby; 1999. p. 209 - 211.

2. Nahmias J, Aurelio J, Gerstein $\mathrm{H}$. An in vitro model for evaluation for electronic root canal length measuring devices. J Endod 1987; 13: 209 $-214$.

3. Walton Torabinejad M. Endodoncia principios y Práctica. 2nda ed. México: Mc Graw-Hill; 1996.

4. McDonald NJ, Hovland EJ. An evaluation of the apex locator Endocater. J Endod 1990; 16: 5 - 8.

5. Kuttler Y. Microscopic investigation of root apexes. J Am Dent Assoc.1955; 50: 544 - 552.

6. Hembrough JH, Weine FS, Pisano JV, Eskoz N. Accuracy of an electronic apex locator: A clinical evaluation in maxillary molars. J Endod 1993; 19: 242 - 246

7. Ibarrola JL, Chapman B, Howard JH, Knowles KI. Effect of preparing on root ZX apex locators. J Endod 1999; 25: 625 - 626.

8. Jenkins JA, Walker WA. An in vitro evaluation of the accuracy of the Root ZX in presence of various irrigants. J Endod 2001; 27: 209 - 211.

9. Imao S. Nuevo método para medir la longitud de trabajo. J Dent Res 1962; $41: 375$ - 387.

10. Shabahang S, Goon WY, Gluxkin AH. An in vivo evaluation of Root ZX electronic apex locator. J Endod 1996; 22: 616 - 617.

11. Pratten DH, McDonald NJ. Comparison of radiographic and electronic working lengths. J Endod.1996; 22: 173-176.

12. Kobayashi C. New electronic canal measuring device based on the ratio method. J Endod 1994; 20: 111 - 114.

13. Frank AL, Torabinejad M. An in vivo evaluation of endex apex locator. J Endod 1993; 19: 177 - 179.

14. Bramamnte C. A critical evaluation of some methods of determining tooth length. Oral Surg Oral Med Oral Pathol 1974; 37: 463 - 469.

15. Weiger R, John X, Geigle H y Löst C. An in vitro comparison of two modern apex locators. J Endod 1999; 25: 765 - 768.

16. Donnelly JC. Simplified model to demonstrate the operation of electronic root canal measuring devices. J Endod.1993; 19: 579 - 580.

17. Kaufman, Katz J. Reliability of root ZX apex locator tested by an in vitro model. J Endod 1993; 19: 20 [Abstract].

18. Ounsi HF, Naaman A. In vitro evaluation of reliability of the Root ZX electronic apex locator. Int J Endod 1999; 32: 120 - 123.

19. Pagano M, Gauvreau K. Principles of biostatistics. Belmont: Duxbury Press; 1993.
20. Bland E, Altman D. Statistical methods for assessing agreement between two methods of clinical measurement. Lancet 1986; 8: 307 310.

21. Ponce E, Fernández JA. The cemento dentino canal - junction, the apical foramen and the apical constriction: Evaluation by optical microscopy. J Endod 2003; 29: 214 - 219.

22. Rivas Muñoz R. Uso y eficacia del localizador del foramen fisiológico, apex finder 7001.URL disponible en: http://www.iztacala.unam.mx/ $\sim$ rrivas/apexfinder.html.

23. Keller ME, Brown CE J, Newton CW. A clinical evaluation of Endocater - An electronic apex locator. J Endod. 1991; 17: 271 - 274.

24. Fouad AF, Rivera EM, Krell KV. Accuracy of endex with variations in canal irrigants and foramen size. J Endod 1993; 19: 63 - 67.

25. Mayeda DL, Simon JH, Aimar DF, Finley K. In vivo measurements accuracy in vital and necrotic canals with the endex apex locator. J Endod 1993; 19: 545 - 548.

26. Kobayashi C. Electronic canal length measurement. Oral Surg Oral Med Oral Pathol 1995; 79: 226 -231.

27. Ounsi HF, Haddad G. In vitro evaluation of the reliability of the endex electronic apex locator. J Endod 1998; 24:120 - 121.

28. Pagavino G, Pace R, Baccetti T. A SEM study of in vivo accuracy of the Root ZX electronic apex locator. J Endod 1998; 24: 438 - 441.

29. Cruz-Filho AM, Sousa-Neto MD, Saquy PC, Pecora JD. Evaluación del efecto del EDTAC, CDTA Y EGTA sobre la microdureza dentinal. J Endod 2001; 27: 183 - 184.

30. Pilot TF, Pitts DL. Determination of impedance changes at varying frequencies in relation to root canal position and irrigant. J Endod 1997; 23: 719 - 724.

31. Tinaz AC, Sevimli LS, Gorgul G, Turkoz EG. The effects of sodium hypochlorite concentrations on the accuracy of an apex locating device. J Endod 2002; 28:160 - 162.

32. Comunicación personal con la Ingeniera Zarith Pachón, Ingeniera Química, Instituto Colombiano de Petróleos (ICP). Piedecuesta, Santander, 11 de mayo de 2003.

106

Ustasalud Odontología 2003; 2: 97 - 106

Gay MM. y col. 